\title{
USING NONSMOOTH ANALYSIS FOR NUMERICAL SIMULATION OF CONTACT MECHANICS
}

\author{
Pierre Alart \\ David Dureisseix \\ and Mathieu Renouf \\ LMGC, University Montpellier 2, CNRS UMR5508, CC048, Place Eugène Bataillon, \\ F-34095 Montpellier Cedex 5, France \\ alart@Imgc.univ-montp2.fr \\ dureisseix@Imgc.univ-montp2.fr \\ renouf@Imgc.univ-montp2.fr
}

Two different approaches for simulation of contact mechanics prob-lems are investigated. First, the equivalence of the Large Time INcre-ment (LATIN) method for unilateral contact problems, in terms of an augmented Lagrangian approach dedicated to nonsmooth problems, is proved (with the non trivial introduction of two constraints: an equal-ity constraint and an inequality-type constraint). Second, the Finite Element Tearing and Interconnecting method for Contact (FETI-C) is interpreted as a projected gradient with projection, acting on the dual problem. An extension to frictional contact is presented in the context of granular media.

\section{Introduction}

In nonsmooth analysis and mechanics, Jean Jacques Moreau proclaims to be firstly a mechanician and a mathematician who develops only what is strictly necessary for mechanical modeling. He was not initially a numerician even if his last works on granular material require efficient computer resources and algorithms. His mathematical tools proved to be very useful in physical / mechanical modeling (friction, plasticity...) and in applied mathematics related to the solution of partial differential equations and in optimization theory. The theory of duality 
in Convex Analysis plays an important role in the mathematical modeling of the aforementioned fields and in the design of efficient solution schemes based, for instance, on augmented Lagrangian functions. But a lot of numerical developments in intensive computational mechanics, when limited to linear large scale problems, did not call for sophisticated notions of Convex Analysis. This research field led to specific concepts already difficult to handle in a linear context (preconditioning, domain decomposition methods...).

The motivation of this work is the re-engineering of algorithms arising from 'intuitive' approaches of computational mechanics, using convex and nonsmooth analysis. This could be a guide for algorithm extensions and development of new approaches.

\section{$1.1 \quad$ Model problem}

To simplify the presentation, let us consider the case of the frictionless Signorini problem depicted in Figure 1.1: only one elastic body $\Omega$ pressed against a rigid plate, under small perturbations, with a quasistatic evolution process. The potential contact area $\Gamma$ will be called an interface. $\underline{n}$ designates the outward unitary normal vector on the boundary $\partial \Omega$.
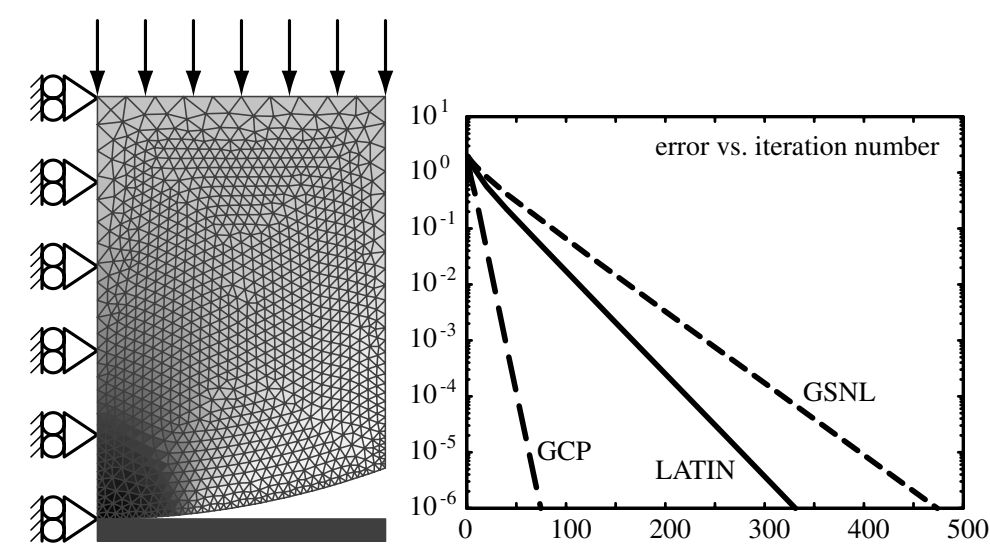

Figure 17.1. Left: vertical stress field solution on the model problem (cylindrical punch); right: convergence results for different iterative algorithms

If $\underline{F}$ is the force applied to the body by the plate, and if $\underline{V}$ is the displacement of the body on the interface $\Gamma$, the interface behavior is: (i) no friction, i.e. $\left(\mathbf{1}-\underline{n n}^{T}\right) \underline{F}=0$, and (ii) complementarity conditions (Karush-Khun-Tucker): 
- no penetration: $z=\underline{n}^{T} \underline{V} \geq 0$, i.e. $z \in \mathcal{C}_{1}$ (the positive cone);

- no adhesion: $w=\underline{n}^{T} \underline{F} \geq 0$, i.e. $w \in \mathcal{C}_{1}$;

- no dissipation: $w z=0$.

The problem consists in finding a displacement field $\underline{U}(M)$, and a stress field $\boldsymbol{\sigma}(M)$, such that:

- $\underline{U}$ is sufficiently regular (the strain field is: $\left.\varepsilon=(\operatorname{grad} \underline{U})_{\text {sym }}\right)$, and equals some prescribed values on a first part of the boundary: $\underline{U}_{\mid \partial_{1} \Omega}=\underline{U}_{d}$, and on the interface: $\underline{n}^{T} \underline{U}_{\mid \Gamma}=z$; a couple $(\underline{U}, z)$ satisfying these conditions is said kinematically admissible (KA);

- the stress field balances the external body force $f_{d}$ and the prescribed forces on a second part of the boundary: $\underline{\operatorname{div}} \boldsymbol{\sigma}+\underline{f}_{d}=0$ in $\Omega, \boldsymbol{\sigma} \underline{n}_{\mid \partial_{2} \Omega}=\underline{F}_{d}$, and on the interface: $\boldsymbol{\sigma} \underline{n}_{\mid \Gamma}=w \underline{n}$; a couple $(\boldsymbol{\sigma}, w)$ satisfying these conditions is said statically admissible (SA);

- the constitutive relation of the body (linear elasticity with $\boldsymbol{D}$ as Hooke tensor): $\boldsymbol{\sigma}=\boldsymbol{D} \boldsymbol{\varepsilon}$, and of the interface (see above).

There are several ways to formulate this problem: minimization with inequality-like constraints, variational inequalities, linear complementarity problems, differential inclusions... The formulations of the next Sections can be derived from the following: the problem is to find $(\underline{U}, z, \boldsymbol{\sigma}, w)$ minimizing the so-called error in constitutive relation $\eta$ for contact problems (Coorevits et al., 1999):

$$
\eta^{2}(\underline{U}, z, \boldsymbol{\sigma}, w)=\frac{1}{2} \int_{\Omega} \operatorname{Tr}\left[(\boldsymbol{\sigma}-\boldsymbol{D} \varepsilon(\underline{U})) \boldsymbol{D}^{-1}(\boldsymbol{\sigma}-\boldsymbol{D} \varepsilon(\underline{U}))\right] d \Omega+\int_{\Gamma} w z d \Gamma
$$

on the set of admissible fields $(\underline{U}, z) \mathrm{KA},(\boldsymbol{\sigma}, w) \mathrm{SA}$, and $z \in \mathcal{C}_{1}, w \in$ $\mathcal{C}_{1}$ (on this set, $\eta^{2}$ is obviously positive, and is zero if and only if the constitutive relations are satisfied).

Using Stoke's theorem, for admissible fields, the expression of $\eta^{2}$ can be split into two uncoupled terms: $\eta^{2}=\eta_{1}^{2}(\underline{U}, z)+\eta_{2}^{2}(\boldsymbol{\sigma}, w)$ with:

$$
\begin{array}{r}
\eta_{1}^{2}(\underline{U}, z)=\frac{1}{2} \int_{\Omega} \operatorname{Tr}[\varepsilon(\underline{U}) \boldsymbol{D} \boldsymbol{\varepsilon}(\underline{U})] d \Omega-\int_{\Omega} \underline{f}_{d} \cdot \underline{U} d \Omega-\int_{\partial_{2} \Omega} \underline{F}_{d} \cdot \underline{U} d S \\
\eta_{2}^{2}(\boldsymbol{\sigma}, w)=\frac{1}{2} \int_{\Omega} \operatorname{Tr}\left[\boldsymbol{\sigma} \boldsymbol{D}^{-1} \boldsymbol{\sigma}\right] d \Omega-\int_{\partial_{1} \Omega} \underline{U}_{d} \cdot \boldsymbol{\sigma} \underline{n} d S
\end{array}
$$

The minimization of the first part only leads to the primal formulation of the problem, while the minimization of the second one leads to the dual (in the Legendre-Fenchel way) formulation. Mixed formulations can be obtained by dualizing each of them (in the Arrow-Hurwicz way) using a Lagrange multiplier (De Saxce, 1989). 


\subsection{Discretized formulations}

The discretized version of the primal formulation, once finite elements are used, is:

$$
\min _{\boldsymbol{B} \boldsymbol{u} \geq 0} \frac{1}{2} \boldsymbol{u}^{T} \boldsymbol{K} \boldsymbol{u}-\boldsymbol{u}^{T} \boldsymbol{f}
$$

where $\boldsymbol{u}$ is the set of finite element degrees of freedom (dof), $\boldsymbol{K}$ is the stiffness matrix, and $\boldsymbol{f}$ are the prescribed generalized forces. $\boldsymbol{B}$ is a Boolean operator that selects the dof on the interface; $\boldsymbol{z}=\boldsymbol{B} \boldsymbol{u} . \quad \boldsymbol{B}$ can also change to the local basis on normal direction; for the sake of simplicity, we assume in all of the following that no such rotations are required. Therefore, the previous problem is easily condensed on the contact dof $\boldsymbol{z}$ and leads to

$$
\min _{\boldsymbol{z} \geq 0} \phi(\boldsymbol{z}) \quad \text { with } \quad \phi(\boldsymbol{z})=\frac{1}{2} \boldsymbol{z}^{T} \boldsymbol{M} \boldsymbol{z}+\boldsymbol{z}^{T} \boldsymbol{q}
$$

where $\boldsymbol{M}$ is the condensed stiffness matrix, and $\boldsymbol{q}$ the opposite of the condensed generalized forces.

Using the indicator function $\psi_{\mathcal{C}_{1}}$ of the discretized cone $\mathcal{C}_{1}$, this is equivalent to:

$$
\min _{\boldsymbol{z}} \phi(\boldsymbol{z})+\psi_{\mathcal{C}_{1}}(\boldsymbol{z}) \quad \Leftrightarrow \quad 0 \in \nabla \phi(\boldsymbol{z})+\partial \psi_{\mathcal{C}_{1}}(\boldsymbol{z})
$$

The problem can therefore be re-stated as to find $\boldsymbol{a} \in \partial \psi_{\mathcal{C}_{1}}(\boldsymbol{z})$ such that $\nabla \phi(\boldsymbol{z})+\boldsymbol{a}=0 . \quad \boldsymbol{a}$ must satisfy: $\psi_{\mathcal{C}_{1}}^{\star}(\boldsymbol{a})+\psi_{\mathcal{C}_{1}}(\boldsymbol{z})-\boldsymbol{a}^{T} \boldsymbol{z} \leq 0$, where $\psi_{\mathcal{C}_{1}}^{\star}$ is the Legendre-Fenchel transform of $\psi_{\mathcal{C}_{1}}$. As the Legendre-Fenchel inequality should be satisfied, $\psi_{\mathcal{C}_{1}}^{\star}(\boldsymbol{a})+\psi_{\mathcal{C}_{1}}(\boldsymbol{z})-\boldsymbol{a}^{T} \boldsymbol{z} \geq 0$, the previous inequality reduces to an equality. This equality is equivalent to: $\boldsymbol{a} \leq 0$, $\boldsymbol{z} \geq 0$ and $\boldsymbol{a}^{T} \boldsymbol{z}=0$. Using $\boldsymbol{w}=-\boldsymbol{a}$, the problem is then to find $(\boldsymbol{z}, \boldsymbol{w})$ such that

$$
\boldsymbol{M} \boldsymbol{z}-\boldsymbol{w}=-\boldsymbol{q} \quad \text { with } \quad 0 \leq \boldsymbol{z} \perp \boldsymbol{w} \geq 0
$$

This is a classical linear complementarity problem (LCP), (Cottle et al., 1992). The unknown is the couple $(\boldsymbol{z}, \boldsymbol{w})$.

A dual formulation can be built from (17.2): with the indicator function $\psi_{\mathcal{C}_{1}}(\boldsymbol{z})=\sup _{\boldsymbol{w}>0}(-\boldsymbol{z})^{T} \boldsymbol{w}$, it is equivalent to: $\inf _{\boldsymbol{z}}\left[\sup _{\boldsymbol{w}>0} \phi(\boldsymbol{z})-\right.$ $\left.\boldsymbol{z}^{T} \boldsymbol{w}\right]$. With sensible assumptions (convexity, semicontinuity) this is again equivalent to: $\sup _{\boldsymbol{w} \geq 0}\left[\inf _{\boldsymbol{z}} \phi(\boldsymbol{z})-\boldsymbol{z}^{T} \boldsymbol{w}\right]$. The inner minimization problem leads to $\boldsymbol{z}=\boldsymbol{M}^{-1}(-\boldsymbol{q}+\boldsymbol{w})$. Substituting this last value in the previous problem leads to the dual problem:

$$
\sup _{\boldsymbol{w} \geq 0}-\frac{1}{2} \boldsymbol{w}^{T} \boldsymbol{M}^{-1} \boldsymbol{w}+\boldsymbol{w}^{T} \boldsymbol{M}^{-1} \boldsymbol{q}
$$




\section{The LATIN method for contact problems}

The LATIN method (Ladevèze, 1999) was originally designed for time-dependent problems. However, it has been applied to different situations, and among them domain decomposition, contact problems (Champaney et al., 1995)... We are interested herein with the version dealing with contact between substructures, for which the method can be viewed as a mixed domain decomposition method, with nonlinear behavior of interfaces. We will focus on frictionless contact. The principles of the LATIN method will be briefly recalled, especially the splitting it introduces for the problem unknowns and constraints, since the augmented Lagrangian approaches are often compared to operator splitting methods (Fortin and Glowinski, 1982, Glowinski and Le Tallec, 1989).

\subsection{The LATIN algorithm}

To simplify the presentation, we will derive all the presentation for the discretized problem (17.3), though the method is directly applicable to the continuum media problem. In each case, the unknowns are both the displacement $\boldsymbol{z}$ and the forces $\boldsymbol{w}$. The LATIN method is based on three mechanical principles:

- splitting the difficulties: the linear equations (possibly global) are collected into the set $\boldsymbol{A}_{d}$, while the local equations (possibly non linear) are collected into the second set $\boldsymbol{\Gamma}$. The solution is the intersection of these sets.

For the problem (17.3), $(\boldsymbol{w}, \boldsymbol{z})$ belongs to $\boldsymbol{A}_{d}$ iff $\boldsymbol{M} \boldsymbol{z}-\boldsymbol{w}=-\boldsymbol{q}$, and $(\hat{\boldsymbol{w}}, \hat{\boldsymbol{z}})$ belongs to $\boldsymbol{\Gamma}$ iff $0 \leq \hat{\boldsymbol{z}} \perp \hat{\boldsymbol{w}} \geq 0$;

- building a 2-stage iterative algorithm: the solution is searched with the construction of two series of approximations belonging alternatively to $\boldsymbol{A}_{d}$ and $\boldsymbol{\Gamma}$.

At iteration $n$, the local stage consists in finding $(\hat{\boldsymbol{w}}, \hat{\boldsymbol{z}}) \in \boldsymbol{\Gamma}$ with a first search direction $\left(\hat{\boldsymbol{w}}-\boldsymbol{w}_{n}, \hat{\boldsymbol{z}}-\boldsymbol{z}_{n}\right) \in \boldsymbol{E}^{+} .\left(\boldsymbol{w}_{n}, \boldsymbol{z}_{n}\right)$ is known at this stage.

The linear stage consists in finding $\left(\boldsymbol{w}_{n+1}, \boldsymbol{z}_{n+1}\right) \in \boldsymbol{A}_{d}$ with a second search direction $\left(\boldsymbol{w}_{n+1}-\hat{\boldsymbol{w}}, \boldsymbol{z}_{n+1}-\hat{\boldsymbol{z}}\right) \in \boldsymbol{E}^{-}$, while $(\hat{\boldsymbol{w}}, \hat{\boldsymbol{z}})$ is known from the previous stage;

- using an ad hoc representation of the unknowns. This last principle is suited to time-dependent problems, and won't be used herein.

The choice for the search direction is classically to use a parameter $k$ homogeneous to an interface stiffness. For the local stage, the search 
direction is: $\left(\hat{\boldsymbol{w}}-\boldsymbol{w}_{n}\right)-k\left(\hat{\boldsymbol{z}}-\boldsymbol{z}_{n}\right)=0$. The local stage is a non-linear and local updating problem, but its solution $(\hat{\boldsymbol{z}}, \hat{\boldsymbol{w}})$ is explicit:

$$
\begin{array}{r}
\hat{\boldsymbol{z}}=-k^{-1}\left\langle\boldsymbol{w}_{n}-k \boldsymbol{z}_{n}\right\rangle_{-} \\
\hat{\boldsymbol{w}}=\left\langle\boldsymbol{w}_{n}-k \boldsymbol{z}_{n}\right\rangle_{+}
\end{array}
$$

$\langle\bullet\rangle_{-}$and $\langle\bullet\rangle_{+}$designate respectively the negative part and the positive part of their argument.

For the linear stage, the unknowns are $\left(\boldsymbol{z}_{n+1}, \hat{\boldsymbol{w}}\right)$ and the conjugate search direction is used: $\left(\boldsymbol{w}_{n+1}-\hat{\boldsymbol{w}}\right)+k\left(\boldsymbol{z}_{n+1}-\hat{\boldsymbol{z}}\right)=0$. The linear stage is a global and linear updating problem: $\boldsymbol{z}_{n+1}$ is obtained with

$$
(\boldsymbol{M}+k \mathbf{1}) \boldsymbol{z}_{n+1}=(\hat{\boldsymbol{w}}+k \hat{\boldsymbol{z}})-\boldsymbol{q} \text { and } \quad \boldsymbol{w}_{n+1}=(\hat{\boldsymbol{w}}+k \hat{\boldsymbol{z}})-k \boldsymbol{z}_{n+1}
$$

Here, $k$ is a parameter of the method. The value of $k$ has no influence on the solution at convergence, but it modifies the convergence rate. Numerical simulations show that there is an optimal value, which is related to the interface operator obtained when condensing the problem on the interface. For the present model problem, this operator is $\boldsymbol{M}$, and $k$ can be diagonal values of $\boldsymbol{M}$, or an average of these.

Figure 1.1 reports convergence results (with an energy-norm error) for different iterative algorithms (CPG: conjugate gradient with projection (Renouf and Alart, 2004), NLGS: non-linear Gauss-Seidel (Moreau, 1999, Jean, 1999)). For this test case, the convergence rate comparison is similar to the one that can be obtained in linear case, for which conjugate gradient has usually a higher convergence rate than LATIN, itself higher than for Gauss-Seidel.

\subsection{Interpretation as a nonsmooth augmented Lagrangian approach}

For perfect interfaces, the LATIN algorithm has been proved to be equivalent to the augmented Lagrangian interpretation (Glowinski and Le Tallec, 1990) of the approach proposed in (Lions, 1990), and more precisely to the so-called 'ALG3' algorithm in (Fortin and Glowinski, 1982), see (Champaney et al., 1995). We proposed herein to prove the equivalence to an augmented Lagrangian algorithm for the case of contact without friction.

Beginning with the minimization problem (17.1), the first step is to split the unknown $\boldsymbol{z}$, using an intermediate unknown $\hat{\boldsymbol{z}}$, an to introduce an equality constraint $\boldsymbol{z}=\hat{\boldsymbol{z}}$, as well as an inequality-like on $\hat{\boldsymbol{z}}$ :

$$
\min _{\boldsymbol{z}=\hat{\boldsymbol{z}}, \hat{\boldsymbol{z}} \geq 0} \phi(\boldsymbol{z})
$$


The second step is to use two multipliers $\boldsymbol{w}_{c}$ and $\hat{\boldsymbol{w}}_{c}$ and convex analysis methodology for dualizing the previous constraints (Rockafellar, 1976). The augmented Lagrangian is obtained with a classical dualization and regularization of the equality constraint, while the MoreauYosida technique is applied to the inequality-like constraint, using a positive parameter $k$ (Yosida, 1964, Moreau, 1965):

$$
\begin{aligned}
& \mathcal{L}\left(\boldsymbol{z}, \hat{\boldsymbol{z}} ; \boldsymbol{w}_{c}, \hat{\boldsymbol{w}}_{c}\right)=\phi(\boldsymbol{z})+ \\
&-\frac{1}{2} \boldsymbol{w}_{c}^{T} k^{-1} \boldsymbol{w}_{c}+\frac{1}{2}\left[\boldsymbol{w}_{c}+k(\boldsymbol{z}-\hat{\boldsymbol{z}})\right]^{T} k^{-1}\left[\boldsymbol{w}_{c}+k(\boldsymbol{z}-\hat{\boldsymbol{z}})\right]+ \\
&-\frac{1}{2} \hat{\boldsymbol{w}}_{c}^{T} k^{-1} \hat{\boldsymbol{w}}_{c}^{T}+\frac{1}{2}\left\langle\hat{\boldsymbol{w}}_{c}+k \hat{\boldsymbol{z}}\right\rangle_{-}^{T} k^{-1}\left\langle\hat{\boldsymbol{w}}_{c}+k \hat{\boldsymbol{z}}\right\rangle_{-}
\end{aligned}
$$

The stationary conditions for this augmented Lagrangian are

$$
\begin{aligned}
\frac{\partial \mathcal{L}}{\partial \boldsymbol{z}} & =0=\boldsymbol{M} \boldsymbol{z}+\boldsymbol{q}+\boldsymbol{w}_{c}+k(\boldsymbol{z}-\hat{\boldsymbol{z}}) \\
\frac{\partial \mathcal{L}}{\partial \boldsymbol{w}_{c}} & =0=-k^{-1}\left\{\boldsymbol{w}_{c}-\left[\boldsymbol{w}_{c}+k(\boldsymbol{z}-\hat{\boldsymbol{z}})\right]\right\} \\
\frac{\partial \mathcal{L}}{\partial \hat{\boldsymbol{z}}} & =0=-\boldsymbol{w}_{c}-k(\boldsymbol{z}-\hat{\boldsymbol{z}})+\left\langle\hat{\boldsymbol{w}}_{c}+k \hat{\boldsymbol{z}}\right\rangle_{-} \\
\frac{\partial \mathcal{L}}{\partial \hat{\boldsymbol{w}}_{c}} & =0=-k^{-1}\left\{\hat{\boldsymbol{w}}_{c}-\left\langle\hat{\boldsymbol{w}}_{c}+k \hat{\boldsymbol{z}}\right\rangle_{-}\right\}
\end{aligned}
$$

One can then remark that for the solution, $\boldsymbol{z}=\hat{\boldsymbol{z}}$ and $\boldsymbol{w}_{c}=\hat{\boldsymbol{w}}_{c}=-\boldsymbol{w}$. Designing an iterative Uzawa-like algorithm consists in expressing the successive stationary conditions with the previous obtained values of the unknowns. With the previous remark, the latest values of $\boldsymbol{z}$ or $\hat{\boldsymbol{z}}$ can be substituted one to the other, and similarly for $\boldsymbol{w}_{c}$ and $\hat{\boldsymbol{w}}_{c}$. The successive steps are the following:

- step 1: assuming that the values $\hat{\boldsymbol{w}}_{c}^{(n)}$ and $\hat{\boldsymbol{z}}^{(n)}$ are the latest ones, the stationary with respect to $\boldsymbol{z}$ gives the next value of $\boldsymbol{z}^{(n+1)}$ :

$$
\boldsymbol{M} \boldsymbol{z}^{(n+1)}+\boldsymbol{q}+k\left(\boldsymbol{z}^{(n+1)}-\hat{\boldsymbol{z}}^{(n)}\right)+\hat{\boldsymbol{w}}_{c}^{(n)}=0
$$

- step 2: with this last updated value $\boldsymbol{z}^{(n+1)}$, the stationary with respect to $\boldsymbol{w}_{c}$ gives the next value of $\boldsymbol{w}_{c}^{(n+1)}$ :

$$
\boldsymbol{w}_{c}^{(n+1)}-\left[\hat{\boldsymbol{w}}_{c}^{(n)}+k\left(\boldsymbol{z}^{(n+1)}-\hat{\boldsymbol{z}}^{(n)}\right)\right]=0
$$

- step 3: with these last values $\boldsymbol{z}^{(n+1)}$ and $\boldsymbol{w}_{c}^{(n+1)}$, the stationary with respect to both $\hat{\boldsymbol{z}}$ and $\hat{\boldsymbol{w}}_{c}$ allows to update their values: first, $-\boldsymbol{w}_{c}^{(n+1)}-k\left(\boldsymbol{z}^{(n+1)}-\hat{\boldsymbol{z}}^{(n+1)}\right)+\left\langle\boldsymbol{w}_{c}^{(n+1)}+k \boldsymbol{z}^{(n+1)}\right\rangle_{-}=0$ 
that leads to

$$
\begin{aligned}
\hat{\boldsymbol{z}}^{(n+1)} & =k^{-1}\left\{\left(\boldsymbol{w}_{c}^{(n+1)}+k \boldsymbol{z}^{(n+1)}\right)-\left\langle\boldsymbol{w}_{c}^{(n+1)}+k \boldsymbol{z}^{(n+1)}\right\rangle_{-}\right\} \\
& =k^{-1}\left\langle\boldsymbol{w}_{c}^{(n+1)}+k \boldsymbol{z}^{(n+1)}\right\rangle_{+}
\end{aligned}
$$

and second, $\hat{\boldsymbol{w}}_{c}^{(n+1)}-\left\langle\boldsymbol{w}_{c}^{(n+1)}+k \boldsymbol{z}^{(n+1)}\right\rangle_{-}=0$

The steps 1 and 2 are the linear stage of the LATIN, while the step 3 is the local stage.

Though other permutations may lead to other augmented Lagrangian versions for nonsmooth problems, the proposed one recovers the LATIN method that appears to be efficient for the simulation of large scale assemblies of deformable parts (Champaney et al., 1997).

\section{Gradient methods for large scale contact problems}

We focuss herein on two kinds of large scale problems. Such large scale problems require sophisticated tools arising from the optimization field, traditionally relying on convex and nonsmooth analysis (Bonnans et al., 2003). The first one is related to finite element simulation of assemblies with a dual domain decomposition method. This method is well suited to problems with a large number of degrees of freedom and a few contact conditions between subdomains. The second one deals with simulation of granular materials and structures: the contact conditions are numerous in this case, between rigid bodies in a large collection.

\subsection{Structural frictionless contact problems}

The FETI-C method has been built as an extension of the Finite Element Tearing and Interconnecting (FETI) method (Farhat and Roux, 1991) for the case of contact without friction between subdomains (Dureisseix and Farhat, 2001). The numerical and parallel scalability (i.e. the ability to converge with a number of iterations depending weakly on the number of subdomains) of the FETI method have been examplified, thanks to the presence of a 'coarse' problem acting on the rigid body displacement of the floating subdomains (subdomains without prescribed displacement). Nevertheless, for simplification, no floating subdomain will be considered here, and only one subdomain is assumed to have an interface with a rigid plate (as for the model problem of Figure 1.1).

The extension to interfaces that exhibit an unilateral contact behavior is performed with the following remarks:

- the procedure should act on admissible reaction forces: $\boldsymbol{w} \geq 0$; 
- the classical residual $\boldsymbol{z}$, which is the displacement jump across the interfaces, should be replaced by the contact residual $\tilde{\boldsymbol{z}}=\langle\boldsymbol{z}\rangle_{-}$to monitor the convergence;

- the 'gluing' condition should act on the active contact interface (defined as the part $\Gamma_{a}$ of $\Gamma$ where $\boldsymbol{w}>0$ ), using an adapted Boolean operator: $\boldsymbol{B} \boldsymbol{w} \boldsymbol{r}= \begin{cases}\boldsymbol{r} & \text { on } \Gamma_{a} \\ 0 & \text { on } \Gamma \backslash \Gamma_{a}\end{cases}$

With $\boldsymbol{B}_{\boldsymbol{w}}^{T}$ as the transpose of the matrix $\boldsymbol{B}_{\boldsymbol{w}}$ for a given $\boldsymbol{w}$, one gets the optimization problem (non quadratic due to the dependence of $\boldsymbol{B} \boldsymbol{w}$ on $\boldsymbol{w})$ :

$$
\min _{\boldsymbol{w} \geq 0} \frac{1}{2} \boldsymbol{w}^{T}\left(\boldsymbol{B}_{\boldsymbol{w}}^{T} \boldsymbol{M}^{-1} \boldsymbol{B} \boldsymbol{w}\right) \boldsymbol{w}-\boldsymbol{w}^{T}\left(\boldsymbol{B}_{\boldsymbol{w}}^{T} \boldsymbol{M}^{-1} \boldsymbol{q}\right)
$$

The straightforward extension of FETI approach consists in applying formally a conjugate gradient algorithm to the problem (17.7) as if this problem is quadratic, driving $\tilde{\boldsymbol{z}}$ to zero, and reusing classical preconditioners. It appears to be scalable for the case where there are floating subdomains, for which an additional projection is used (Dureisseix and Farhat, 2001). Of course, the conjugating property is not satisfied for all of the previous search directions because the $\boldsymbol{B}_{\boldsymbol{w}}$ operator projects the iterate, and the $\boldsymbol{B}_{\boldsymbol{w}}^{T}$ operator projects the gradient.

Therefore, this algorithm can be interpreted as a projected conjugate gradient with projection, applied to the classical quadratic dual problem as expressed in (17.4). Work is in progress on adapting FETI-DP version (Farhat et al., 2001) to contact and friction. Such an adaptation would take advantage of the aforementioned interpretation, and of the developments presented in the next Section.

\subsection{Granular frictional contact problems}

Independently, P. Alart and M. Renouf developed a conjugate gradient type algorithm for granular media characterized with a large number of bodies and consequently of contacts (Renouf and Alart, 2003).

A granular material is by essence a discrete medium, where each 'subdomain' is a rigid body. The problem is therefore stated from the point of view of interactions between these bodies. As an example, Figure 3.2 illustrates the simulation of a sandbox experiment, useful in geomechanics for soil layer formation. About 40000 grains in interaction (involving 88000 contact conditions) are modeled.

Each interaction, as an 'interface', deals with the relative velocity between bodies, $\boldsymbol{z}=\boldsymbol{B} \boldsymbol{v}$ (where $\boldsymbol{v}$ stores the velocities of the bodies), as well as the contact impulsion $\boldsymbol{w}$. This is of course a time-dependent 


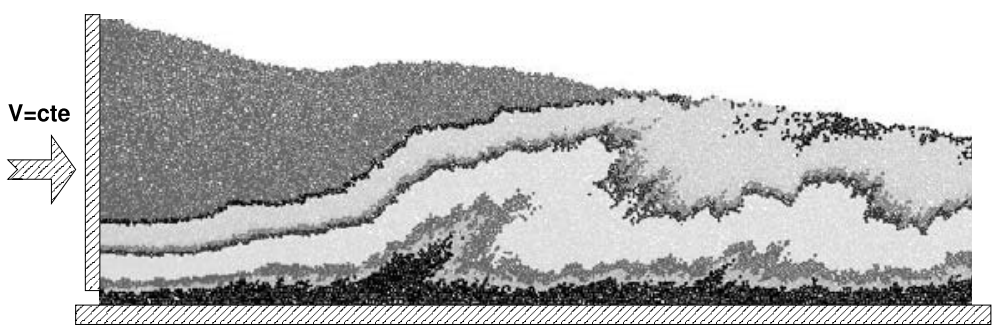

Figure 17.2. Sandbox simulation (40000 grains)

problem, and for a given time step, the dynamic evolution of the bodies is $\boldsymbol{M} \boldsymbol{v}=\boldsymbol{B}^{T} \boldsymbol{w}-\boldsymbol{q}$ where $\boldsymbol{q}$ stores external impulsions and contributions of relative velocities at the end of the previous time step. $\boldsymbol{M}$ is in this case the block-diagonal mass matrix of all the bodies.

For the case of frictionless interaction, the constitutive relations are simply $0 \leq \boldsymbol{z} \perp \boldsymbol{w} \geq 0$. Expressing $\boldsymbol{v}$ from the dynamic evolution, one gets $\boldsymbol{z}=\boldsymbol{W} \boldsymbol{w}-\widetilde{\boldsymbol{q}}$, with $\boldsymbol{W}=\boldsymbol{B} \boldsymbol{M}^{-1} \boldsymbol{B}^{T}$ and $\widetilde{\boldsymbol{q}}=\boldsymbol{B} \boldsymbol{M}^{-1} \boldsymbol{q}$. The resulting problem is therefore similar to the previous ones.

For the case of frictional contact, normal and tangential components at each node have to be separated, and we will use lowerscript $n$ and $t$ to designate them. For simplicity, we will consider only bidimensional analysis. The constraints on impulsion $\boldsymbol{w}$ must belong to the Coulomb's friction cone, i.e. $\boldsymbol{w} \in \mathcal{C}\left(\mu \boldsymbol{w}_{n}\right)$, with $\mu$ as the Coulomb's friction coefficient, $\boldsymbol{w}_{n}$ the normal component of the impulsion, and

$$
\mathcal{C}\left(\mu \boldsymbol{w}_{n}\right)=\prod_{\alpha=1}^{n_{c}} \mathbb{R}^{+} \times\left[-\mu \boldsymbol{w}_{\alpha, n}, \mu \boldsymbol{w}_{\alpha, n}\right]
$$

where $n_{c}$ is the number of potential contact nodes. As for the frictionless contact, the matrix $\boldsymbol{W}=\boldsymbol{B} \boldsymbol{M}^{-1} \boldsymbol{B}^{T}$ is semi definite positive and therefore can be singular. The resulting quasi-optimization problem is:

$$
\boldsymbol{w} \in \underset{\boldsymbol{x} \in \mathcal{C}\left(\mu \boldsymbol{w}_{n}\right)}{\operatorname{argmin}} \frac{1}{2} \boldsymbol{x}^{T} \boldsymbol{W} \boldsymbol{x}-\boldsymbol{x}^{T} \widetilde{\boldsymbol{q}}
$$

The solving procedure relies on a fixed point method for the Tresca threshold $\mu \boldsymbol{w}_{n}$ and on an inner conjugate projected gradient. The proposed approach is a diagonalized version of this procedure (with a one shot single loop). The projection operators are replaced by specific corrections which are not exactly projections, but may be derived from a mathematical analyses in such a way that the converged solution satisfies the relation (17.8), (Renouf and Alart, 2004). 
Figure 17.2 is related to the first time step of the compaction under gravity of a box filled with 33000 grains. The convergence of 3 different algorithms (CPG: conjugate projected gradient, NLGS: non-linear Gauss-Seidel, and PCPG: preconditioned conjugate projected gradient) is compared for increasing values of Coulomb's friction coefficient $\mu$. For all of them, the convergence requires more iterations when friction increases. The conjugate gradient methods are more efficient than the Gauss-Seidel one, but the gain decreases as the friction increases.
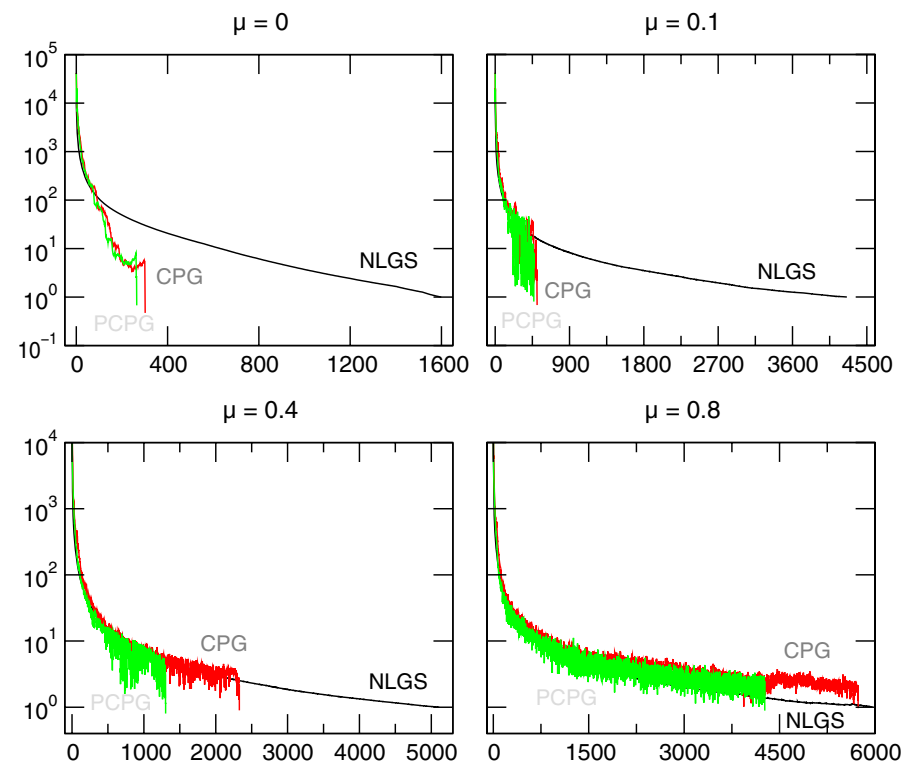

Figure 17.3. Influence of friction coefficient on convergence

\section{Structural versus granular}

Different scientific communities usually develop different dedicated tools that can sometimes be re-unified. Such a gap bridging is done in Section 2.2 where the LATIN algorithm for frictionless contact is reinterpreted as a Lagrangian approach for nonsmooth problems. Convergence results in this case can be transferred to Lagrangian approches as convergence is proved (Ladevèze, 1999) for $0<k<\infty$. The construction of the equivalence can also lead to other versions of the method.

Moreover, between structural analysis and granular simulation communities, the approach used for frictional contact and gradient-based simulations of a large number of grains can guide the development of extensions to frictional case for dual domain decomposition methods. The 
primal domain decomposition method already got benefits from convex and nonsmooth analysis (Alart et al., 2004).

\section{References}

Alart, P., Barboteu, M., and Renouf, M. (2004). Parallel computational strategies for multicontact problems: Applications to cellular and granular media. Int. J. for Multiscale Comput. Engng. to appear.

Bonnans, J.-F., Gilbert, J.-C., Lemaréchal, C., and Sugastizàbal, C. (2003). Numerical Optimization - Theoretical and Practical Aspect. Springer Verlag.

Champaney, L., Cognard, J.-Y., Dureisseix, D., and Ladevèze, P. (1995). Une approche modulaire pour l'analyse d'assemblages de structures tridimensionnelles. Application au contact avec frottement. In Proceedings of StruCoMe 95, pages 295-306, Paris.

Champaney, L., Cognard, J.-Y., Dureisseix, D., and Ladevèze, P. (1997). Large scale applications on parallel computers of a mixed domain decomposition method. Comput. Mech., (19):253-263.

Coorevits, P., Hild, P., and Pelle, J.-P. (1999). Contrôle des calculs éléments finis pour les problèmes de contact unilatéral. Revue Européenne des Éléments Finis, 8(1):7-29.

Cottle, R. W., Pang, J.-S., and Stone, R. E. (1992). The Linear Complementarity Problem. Academic Press, Boston.

De Saxce, G. (1989). Sur quelques problèmes de mécanique des solides considérés comme matériaux à potentiel convexe. volume 118 of $P u$ blications de la Faculté des Sciences appliquées. Université de Liège.

Dureisseix, D. and Farhat, C. (2001). A numerically scalable domain decomposition method for the solution of frictionless contact problems. Int. J. for Numer. Meth. Engng., 50:2643-2666.

Farhat, C., Lesoinne, M., Le Tallec, P., Pierson, K., and Rixen, D. (2001). FETI-DP: a dual-primal unified FETI method - part I: a faster alternative to the two-level FETI method. Int. J. for Numer. Meth. Engng., 50(7):1523-1544.

Farhat, C. and Roux, F.-X. (1991). A method of finite element tearing and interconnecting and its parallel solution algorithm. Int. J. for Numer. Meth. Engng., 32:1205-1227.

Fortin, M. and Glowinski, R. (1982). Méthodes de lagrangien augmenté. In Lions, P.-L., editor, Méthodes Mathématiques de l'informatique, volume 9.

Glowinski, R. and Le Tallec, P. (1989). Augmented lagrangian and operator-splitting methods in nonlinear mechanics. volume 9 of SIAM Studies in Appl. Math. SIAM. 
Glowinski, R. and Le Tallec, P. (1990). Augmented lagrangian interpretation of the nonoverlapping Schwarz alternating method. In Chan, T. F., Glowinski, R., Périaux, J., and Widlund, O. B., editors, Third Int. Symp. on Domain Decomposition Methods for Part. Differ. Equations, pages 224-231. SIAM.

Jean, M. (1999). The non-smooth contact dynamics method. Comput. Meth. Appl. Mech. Engng., 117(3-4):235-257.

Ladevèze, P. (1999). Nonlinear Computational Structural Mechanics New Approaches and Non-Incremental Methods of Calculation. Springer Verlag.

Lions, P.-L. (1990). On the Schwarz alternating method III: a variant for nonoverlapping subdomains. In Chan, T. F., Glowinski, R., Périaux, J., and Widlund, O. B., editors, Third Int. Symp. on Domain Decomposition Methods for Partial Differential Equ., pages 202-223. SIAM.

Moreau, J. J. (1965). Proximité et dualité dans un espace hilbertien. Bulletin de la Société Mathématique de France, 93:273-299.

Moreau, J. J. (1999). Numerical aspects of the sweeping process. Comput. Meth. Appl. Mech. Engng., 117(3-4):329-349.

Renouf, M. and Alart, P. (2003). Un nouvel algorithme de quasi-optimisation pour la résolution des problèmes multicontacts et application aux milieux granulaires. In Proc. of the 16e Congrès Français de Mécanique, Nice.

Renouf, M. and Alart, P. (2004). Conjugate gradient type algorithms for frictional multicontact problems: applications to granular materials. Comput. Meth. Appl. Mech. Engng. submitted.

Rockafellar, R. T. (1976). Augmented lagrangians and applications of the proximal point algorithm in convex programming. Math. of $O p$ erations Research, 1(2):97-116.

Yosida, K. (1964). Functional analysis. Springer Verlag, Berlin. 